\title{
Through-the-mesh technique after endoscopic ultrasonography-guided hepaticogastrostomy: a novel re-intervention method
}
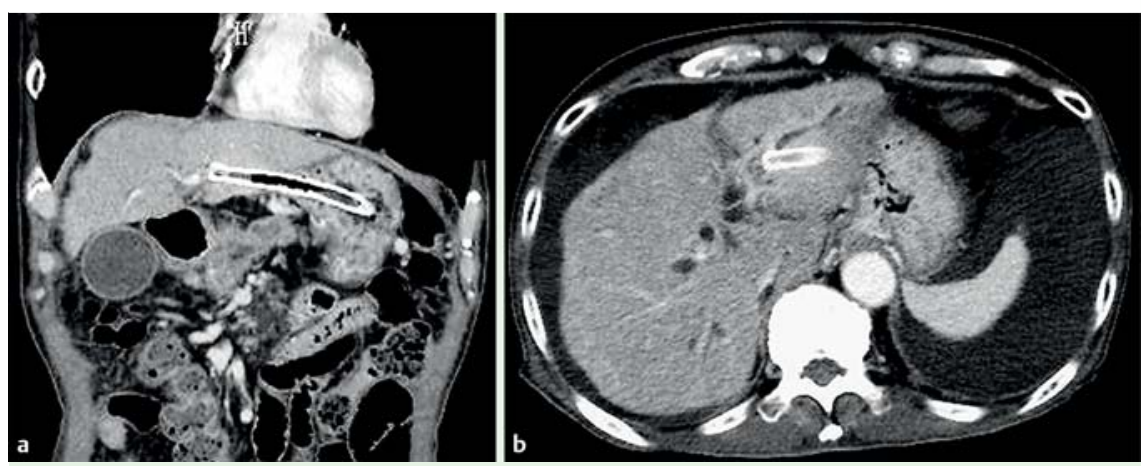

Fig. 1 Computed tomography (CT) images showing: a a covered metal stent deployed between the left intrahepatic bile duct and the stomach; $\mathbf{b}$ a dilated intrahepatic bile duct.
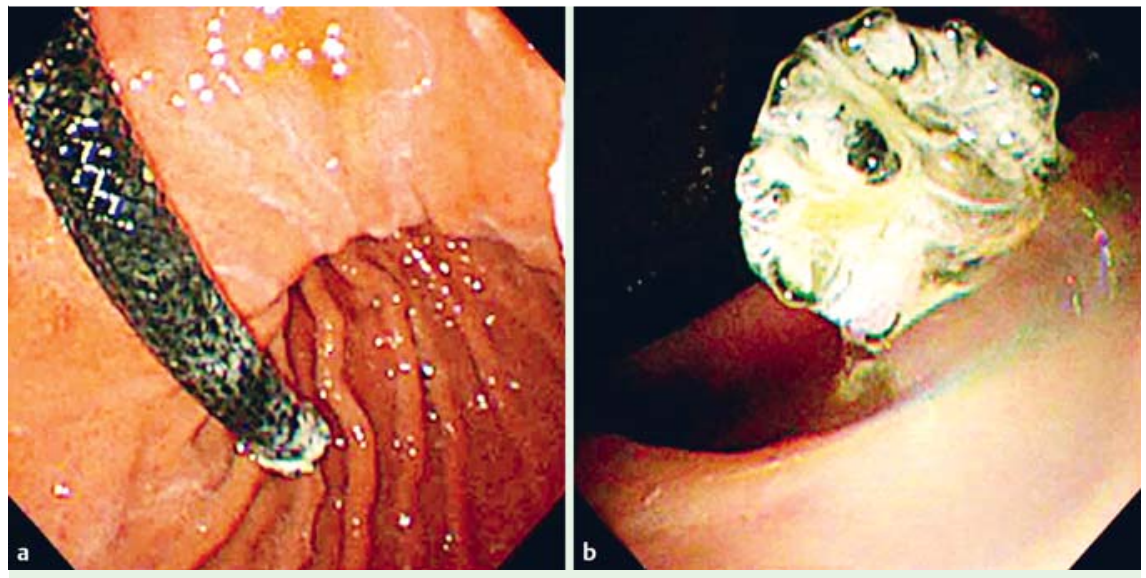

Fig. 2 Gastroscopy showing the occluded metal stent. The length of stent in the gastric lumen was about $5 \mathrm{~cm}$.

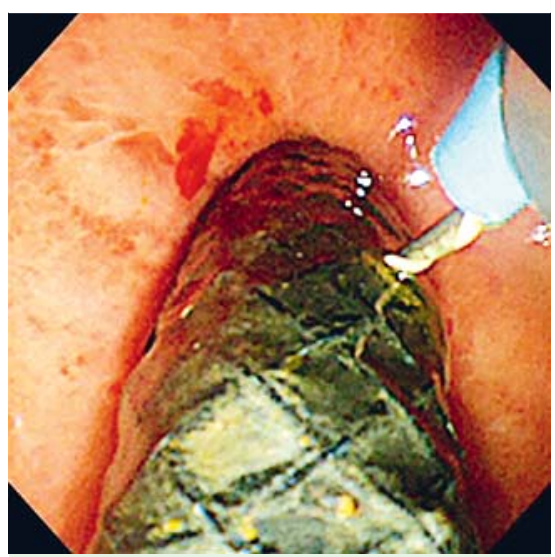

Fig. 3 A 0.025-inch stiff guidewire was inserted to penetrate the stent cover membrane near the gastric puncture site.

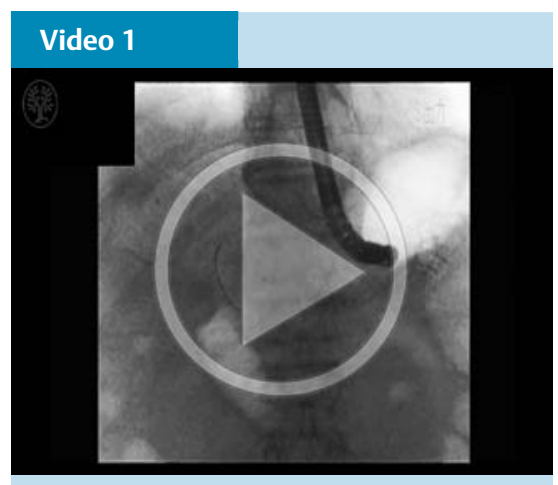

An additional metal stent was deployed through the mesh of the previously placed hepaticogastrostomy (HGS) stent to cover the malignant distal biliary stricture in an antegrade fashion as treatment for HGS stent occlusion.
Endoscopic ultrasonography-guided hepaticogastrostomy (EUS-HGS) is increasingly used to manage failed endoscopic biliary drainage in patients with malignant biliary obstruction [1-3]. A recent study showed that, for EUS-HGS, a stent $\geq 3 \mathrm{~cm}$ in length in the luminal portion may be suitable to prevent stent migration and achieve long-term stent patency [4]. However, such a placement can sometimes make re-intervention difficult. Here, we describe a novel re-intervention technique for HGS stent occlusion.

A 75-year-old man with advanced pancreatic cancer presented with a recurrence of jaundice 11 months after undergoing EUS-HGS using a covered metal stent (Niti-S Biliary Covered Stent; $8 \times 100 \mathrm{~mm}$; Taewoong Medical, Seoul, Korea) for distal malignant biliary obstruction ( $\bullet$ Fig. 1 a). Computed tomography (CT) revealed a dilated intrahepatic and extrahepatic bile duct ( Fig.1 b), and gastroscopy confirmed stent occlusion ( $\bullet$ Fig. 2). Re-intervention was attempted via the HGS route; however, insertion of an endoscopic retrograde cholangiopancreatography (ERCP) catheter into the intrahepatic bile duct through the proximal end of the HGS stent failed.

Re-intervention through the stent mesh was then attempted. A 0.025-inch stiff guidewire was inserted, penetrating the stent cover membrane close to the gastric puncture site ( Fig.3). Next, a $6-\mathrm{mm}$ fine-gauge balloon catheter (REN; 3-Fr tip; Kaneka Medix, Osaka, Japan) was inserted into the bile duct, breaking through and opening the stent cover membrane. After successfully advancing the guidewire through the distal biliary stricture into the duodenum, an additional metal stent (BileRush selective; $10 \times 60 \mathrm{~mm}, 5.7-\mathrm{Fr}$ delivery system; Piolax, Kanagawa, Japan) was inserted through the mesh of the HGS stent to cover the biliary stricture in an antegrade fashion ( $\bullet$ Fig. 4; $\bullet$ Video 1 ).

The postoperative period was uneventful and the patient's jaundice resolved in a few days. This "through-the-mesh" technique is simple and safe, and could be a useful re-intervention option after EUSHGS.

Endoscopy_UCTN_Code_CPL_1AL_2AD

Competing interests: None 

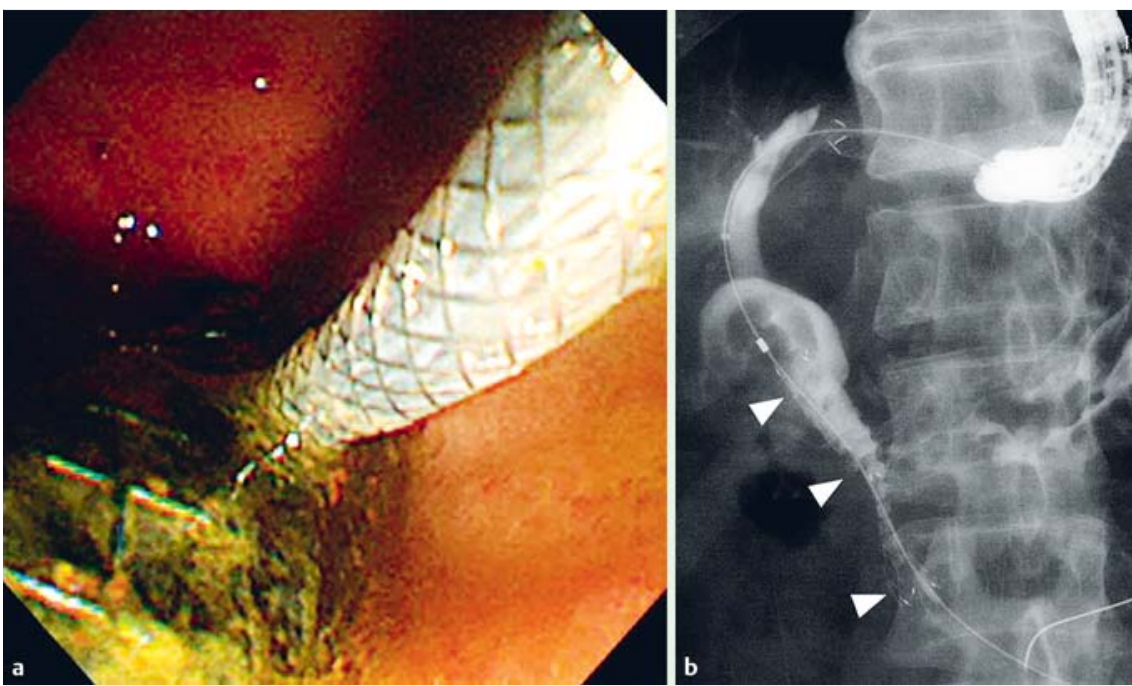

Fig.4 An additional uncovered metal stent with a fine-gauge delivery system that was inserted to cover the distal biliary stricture in an antegrade fashion through the mesh of the previously deployed stent: a in endoscopic view; $\mathbf{b}$ on fluoroscopy.

\section{Kosuke Minaga', Mamoru Takenaka', Takeshi Miyata', Yasuhiro Ueda', Masayuki Kitano ${ }^{2}$, Masatoshi Kudo ${ }^{1}$}

${ }^{1}$ Department of Gastroenterology and Hepatology, Kindai University Faculty of Medicine, Osaka-Sayama, Japan

2 Second Department of Internal Medicine, Wakayama Medical University School of Medicine, Wakayama, Japan

\section{References}

1 Burmester E, Niehaus J, Leineweber T et al. EUS-cholangio-drainage of the bile duct: report of 4 cases. Gastrointest Endosc 2003; 57: $246-251$

2 Poincloux L, Rouquette O, Buc E et al. Endoscopic ultrasound-guided biliary drainage after failed ERCP: cumulative experience of 101 procedures at a single center. Endoscopy 2015; 47: $794-801$
3 Wang $K$, Zhu J, Xing $L$ et al. Assessment and safety of EUS-guided biliary drainage: a systematic review and meta-analysis. Gastrointest Endosc 2016; 83: 1218-1227

4 Ogura T, Yamamoto $K$, Sano $T$ et al. Stent length is impact factor associated with stent patency in endoscopic ultrasound-guided hepaticogastrostomy. J Gastroenterol Hepatol 2015; 30: 1748 - 1752

\section{Bibliography}

DOI http://dx.doi.org/

$10.1055 / \mathrm{s}-0042-117714$

Endoscopy 2016; 48: E369-E370

(c) Georg Thieme Verlag KG

Stuttgart · New York

ISSN 0013-726X

\section{Corresponding author}

Department of Gastroenterology and Hepatology Kindai University Faculty of Medicine 377-2 Ohno-Higashi

Osaka-Sayama, 589-8511

Japan

Fax: +81-72-3672880

mamoxyo45@gmail.com 